П. КУКК, Х. ААРНА, Маре АЛТОСААР, Я. МЯДАССОН, Э. МЕЛЛИКОВ

\title{
ОПРЕДЕЛЕНИЕ КВАЗИХИМИЧЕСКИХ ПАРАМЕТРОВ ТОЧЕЧНЫХ ДЕФЕКТОВ В ПОЛУПРОВОДНИКОВЫХ СОЕДИНЕНИЯХ
}

\author{
(Представил А. Аарна)
}

Проблема дефектообразования и влияния дефектов на свойства кристаллов - одна из центральных в химии твердого тела - приобретает особую актуальность при синтезе полупроводниковых кристаллов, огличающихся высокой чувствительностью к природе и концентрации точечных дефектов [1, 2]. Поэтому прогнозирование структурно-чувствительных свойств полупроводниковых материалов и реализация их направленным дефектообразованием базируется на физико-химических исследованиях по схеме синтез-дефектный состав-свойство $\left.{ }^{2-4}\right]$.

В настоящей работе излагается новая методика расчета квазихимических параметров - показателей степеней концентрации дефектов в зависимости от препаративных условий (давления паров компонента, концентрации легирующих примесей), служащих основой для нахождения корреляционной связи между синтезом (препаративными условиями) и дефектным составом полупроводниковых твердых фаз переменного состава. Предлагаемая методика расчета квазихимических параметров является дальнейшим развитием представлений, выдвинутых в $[5,6]$.

Для конкретности рассмотрим полупроводниковое соединение класса $\mathrm{A}^{\mathrm{II}} \mathrm{B}^{\mathrm{VI}}$ - сульфид кадмия, легированный хлором и медью, наиболее эффективными донорной и акцепторной примесями для этого класса полупроводников $\left[{ }^{7,8}\right]$. Необходимые для квазихимического анализа уравнения закона действующих масс квазихимических реакций дефектообразования (КХРД) для $\mathrm{CdS}: \mathrm{Cl}: \mathrm{Cu}$ приведены в табл. 1 . К уравнениям равновесия КХРД (табл. 1) добавляются уравнение электронейтральности (УЭН)

$$
\begin{gathered}
n+2\left[V_{\mathrm{Cd}}^{\prime \prime}\right]+\left[V_{\mathrm{Cd}}^{\prime}\right]+\left[V_{\mathrm{Cd}}^{\prime \prime} \mathrm{Cl}_{\mathrm{s}}^{\cdot}\right]+\left[\mathrm{Cu}_{\mathrm{Cd}}^{\prime}\right]+2\left[\left(\mathrm{Cu}_{\mathrm{Cd}}\right)_{2}^{\prime}\right]= \\
=p \cdot+2\left[V_{\mathrm{s}}^{*}\right]+\left[V_{\mathrm{s}}^{\cdot}\right]+\left[\mathrm{Cl}_{\mathrm{s}}\right]+\left[\mathrm{Cu}_{i}^{\cdot}\right]
\end{gathered}
$$

и уравнения материального баланса (УМБ) по хлору

$$
\begin{gathered}
{\left[\mathrm{Cl}_{\mathrm{S}}\right]+\left[\mathrm{V}_{\mathrm{Cd}}^{\prime \prime} \mathrm{Cl}_{\mathrm{s}}\right]+2\left[\mathrm{~V}_{\mathrm{Cd}}^{\prime \prime}\left(\mathrm{Cl}_{\mathrm{S}}^{-}\right)_{2}\right]+\left[\mathrm{Cu}_{\mathrm{Cd}}^{\prime} \mathrm{Cl}_{\mathrm{S}}^{-}\right]+} \\
+2\left[\left(\mathrm{Cu}_{\mathrm{Cd}}^{\prime} \mathrm{Cl}_{\mathrm{S}}^{-}\right)_{2}\right]=[\mathrm{Cl}]
\end{gathered}
$$

и меди

$$
\begin{gathered}
{\left[\mathrm{Cu}_{\mathrm{Cd}}^{\prime}\right]+\left[\mathrm{Cu}_{i}^{\cdot}\right]+2\left[\mathrm{Cu}_{\mathrm{Cd}}^{\prime} \mathrm{Cu}_{i}^{\cdot}\right]+4\left[\left(\mathrm{Cu}_{\mathrm{Cd}}^{\prime} \mathrm{Cu}_{i}^{\cdot}\right)_{2}\right]+} \\
+\left[\mathrm{Cu}_{\mathrm{Cd}}^{\prime} \mathrm{Cl}_{\mathrm{S}}^{\cdot}\right]+2\left[\left(\mathrm{Cu}_{\mathrm{Cd}}^{\prime} \mathrm{Cl}_{\mathrm{S}}^{\cdot}\right)_{2}\right]=[\mathrm{Cu}] .
\end{gathered}
$$


Можно выделить при этом два простых случая.

A. Количество хлора и меди выше предела растворимости этих примесей в сульфиде кадмия. Тогда химический состав системы $\mathrm{CdS}: \mathrm{Cl}: \mathrm{Cu}$ однозначно определяется $T$ (температурой термообработки) и $p_{\mathrm{Cd}}$ (давлением паров кадмия). Этот случай рассмотрен в [5].

Б. Количество хлора и меди ниже предела растворимости этих примесей в сульфиде кадмия. Тогда химический состав системы $\mathrm{CdS}: \mathrm{Cl}: \mathrm{Cu}$ определяется $T, p_{\mathrm{Cd}},[\mathrm{Cl}]$ и $[\mathrm{Cu}]([\mathrm{Cl}],[\mathrm{Cu}]-$ общие растворенные концентрации хлора и меди в сульфиде кадмия). Этот последний случай будет рассмотрен ниже.

Решив систему уравнений, включающую уравнения равновесия КХРД (табл. 1), кроме реакций растворения примесей, УМБ (2) и (3), предварительно упрощенные приближением Броуэра [ $\left.{ }^{9}\right]$, при парамет$\operatorname{pax} p_{\mathrm{Cd}}, n$ (концентрация свободных электронов) и [Cu], получаем

$$
\left[X_{m g}^{g}\right]=K_{m}^{g} p_{\mathrm{Cd}}^{\sigma_{m g}} n^{\eta_{m g}}[\mathrm{Cl}]^{\tau_{m g}}[\mathrm{Cu}]^{\hat{\theta}_{m g}},
$$

где $K_{m}^{g}-$ коэффициент, зависящий только от $T$. Учитывая степенную зависимость концентрации дефектов в броуэровском приближении, в частности свободных электронов от $p_{\mathrm{Cd}}$, [Cl] и $[\mathrm{Cu}][2,3,9-12]$, получаем

$$
n \sim p_{\mathrm{Cd}}^{\gamma_{n}}[\mathrm{Cl}]^{\alpha_{n}}[\mathrm{Cu}]^{\beta_{n}}
$$

Подставляя (5) в (4), получаем для квазихимических параметров дефекта $X_{m}^{g}$

$$
\begin{aligned}
& \gamma_{m g}=\sigma_{m g}+\gamma_{n} \eta_{m g}, \\
& \alpha_{m g}=\tau_{m g}+\alpha_{n} \eta_{m g}, \\
& \beta_{m g}=\vartheta_{m g}+\beta_{n} \eta_{m g},
\end{aligned}
$$

где $\gamma_{m g}, \alpha_{m g}, \beta_{m g}-$ показатели степени в функциях $\left[X_{m}^{g}\right]$ от $p_{\mathrm{C} d}$, $[\mathrm{Cl}]$ и $[\mathrm{Cu}]$ соответственно. Значения $\gamma_{n}, \alpha_{n}$ и $\beta_{n}$ определяют экспериментально, измерив зависимость высокотемпературной проводимости от давления паров компонента и содержания примесей. Анализ (6)-(8) на основе табл. 1 и 2 показывает, что значения $\sigma_{m g}, \tau_{m g}$ и $\theta_{m g}$ не зависят от зарядового состояния дефекта, следовательно, индекс $g$ может быть опущен; $\eta_{m g}$ равно эффективному заряду дефекта с обратным знаком

$$
\eta_{m g}=-g \text {. }
$$

Таким образом, уравнения (6)-(8) могут быть представлены в более простом виде

$$
\begin{aligned}
& \gamma_{m g}=\sigma_{m}-\gamma_{n g}, \\
& \alpha_{m g}=\tau_{m}-\alpha_{n g}, \\
& \beta_{m g}=\vartheta_{m}-\beta_{n g} .
\end{aligned}
$$

При выводе $(6)-(8)$ учтен тот факт, что электрически активные примеси в соединениях $\mathrm{A}^{\text {I }}{ }^{\mathrm{VI}}$ растворяются преимущественно в электронейтральной форме $\left[{ }^{13-15}\right]$, т. е в УМБ (2) и (3) доминирует один из электронейтральных дефектов. В противном случае $\eta_{m g}$ в (7) и (8) нельзя было бы представить равенством (9).

Уравнения (10)-(12) легли в основу новой методики определения квазихимических параметров $\gamma_{m g}, \alpha_{m g}, \beta_{m g}$ точечных дефектов $X_{m}^{g}$ и выбора доминирующих дефектов в твердых телах. Для дефек- 


$$
\begin{aligned}
& \mathrm{CdS}=\mathrm{Cd}(g)+1 / 2 \mathrm{~S}_{2}(g) \\
& \mathrm{Cd}(g)=V \cdot{ }_{\mathrm{s}}+2 e^{\prime}+\mathrm{Cd}_{\mathrm{Cd}}^{x} \\
& 0=V \cdot \cdot \overrightarrow{\mathrm{s}}+V_{\mathrm{Cd}}^{\prime \prime} \\
& 0=e^{\prime}+h^{\prime} \\
& V_{\mathrm{s}}^{x}=V_{\mathrm{s}}^{\cdot}+e^{\prime} \\
& V_{\mathrm{s}}=V_{\mathrm{s}}+e^{\prime} \\
& V_{\mathrm{Cd}}^{x}=V_{\mathrm{Cd}}^{\prime}+h^{.} \\
& V_{\mathrm{Cd}}^{\prime \prime}=V_{\mathrm{Cd}}^{\prime}+e^{\prime} \\
& 0=V_{\mathrm{Cd}}^{x}+V_{\mathrm{s}}^{x} \\
& \mathrm{CdCl}_{2}(l)=V_{\mathrm{Cd}}^{\prime \prime}\left(\mathrm{Cl}_{\mathrm{S}}^{\cdot}\right)_{2}+\mathrm{Cd}^{x} \\
& 1 / 2 \mathrm{CdCl}_{2}(g)+\mathrm{Cd}(g)=\mathrm{Cd}_{\mathrm{Cd}}^{x}+\left(\mathrm{Cl}_{\mathrm{s}} \cdot\right)+e^{\prime} \\
& \mathrm{Cl}_{\mathrm{s}}^{x}=\mathrm{Cl}_{\mathrm{s}}+e^{\prime} \\
& \mathrm{Cl}_{\mathrm{s}}+\mathrm{V}_{\mathrm{Cd}}^{\prime \prime}=\mathrm{V}_{\mathrm{Cd}}^{\prime \prime} \mathrm{Cl}_{\mathrm{s}} \\
& \mathrm{Cl}_{\mathrm{s}}+V_{\mathrm{Cd}}^{\prime \prime}\left(\mathrm{Cl}_{\mathrm{s}}^{\cdot}\right)=V_{\mathrm{Cd}}^{\prime \prime}\left(\mathrm{Cl}_{\mathrm{s}}^{\cdot}\right)_{2} \\
& 2 \mathrm{Cu}_{2} \mathrm{~S}(s)=\left(\mathrm{Cu}_{\mathrm{Cd}} \mathrm{Cu}_{i}\right){ }_{2}^{x}+2 \mathrm{~S}_{\mathrm{S}}^{x} \\
& \mathrm{Cu}(s)+\mathrm{Cd}_{\mathrm{Cd}}^{x}=1 / 2\left(\mathrm{Cu}_{\mathrm{Cd}} \mathrm{Cu}_{i}\right)^{x}+\mathrm{Cd}(g) \\
& \left(\mathrm{CdCl}_{2}+\mathrm{Cu}_{2} \mathrm{~S}\right)(l)=2\left(\mathrm{Cu}_{\mathrm{Cd}} \mathrm{Cl}_{\mathrm{S}}\right)^{x}+\mathrm{Cd}_{\mathrm{Cd}}^{x}+\mathrm{S}_{\mathrm{S}}^{x} \\
& \left(\mathrm{Cu}_{\mathrm{Cd}} \mathrm{Cu}_{i}\right)^{x}=\mathrm{Cu}_{\mathrm{Cd}}^{\prime}+\mathrm{Cu}_{i} \\
& 2\left(\mathrm{Cu}_{\mathrm{Cd}} \mathrm{Cu}_{i}\right)^{x}=\left(\mathrm{Cu}_{\mathrm{Cd}} \mathrm{Cu}_{i}\right)_{2}^{x} \\
& \mathrm{Cu}_{\mathrm{Cd}}^{\prime}=V_{\mathrm{Cd}}^{\prime \prime}+\mathrm{Cu}_{i}{ }_{i} \\
& \mathrm{Cu}_{\mathrm{Cd}}^{x}=\mathrm{Cu}_{\mathrm{Cd}}^{\prime}+h \text {. } \\
& \mathrm{Cu}_{i}^{x}=\mathrm{Cu}_{i}^{*}+e^{\prime} \\
& 2 \mathrm{Cu}_{\mathrm{Cd}}^{\prime}=\left(\mathrm{Cu}_{\mathrm{Cd}}^{\prime}\right)_{2} \\
& \mathrm{Cu}_{\mathrm{Cd}}^{\prime}+\mathrm{Cl}_{\mathrm{s}}^{\cdot}=\left(\mathrm{Cu}_{\mathrm{Cd}}^{\prime} \mathrm{Cl}_{\mathrm{s}}^{\cdot}\right)^{x} \\
& 2\left(\mathrm{Cu}_{\mathrm{Cd}} \mathrm{Cl}_{\mathrm{s}}\right)^{x}=\left(\mathrm{Cu}_{\mathrm{Cd}} \mathrm{Cl}_{\mathrm{S}}\right)_{2}^{x} \\
& V_{\mathrm{Cd}}^{\prime \prime}\left(\mathrm{Cl}_{\mathrm{S}}^{\cdot}\right)_{2}+\mathrm{Cu}_{\mathrm{Cd}}^{\prime}=\mathrm{Cu}_{\mathrm{Cd}}^{\prime} \mathrm{Cl}_{\mathrm{S}}^{\cdot}+\mathrm{V}_{\mathrm{Cd}}^{\prime \prime} \mathrm{Cl}_{\mathrm{S}} \\
& \mathrm{Cu}_{i}+V_{\mathrm{Cd}}^{\prime \prime} \mathrm{Cl}_{\mathrm{s}}=\mathrm{Cu}_{\mathrm{Cd}}^{\prime} \mathrm{Cl}_{\mathrm{s}} \text {. } \\
& \left(\mathrm{Cu}_{\mathrm{Cd}} \mathrm{Cu}_{i}\right)_{2}^{x}=2\left(\mathrm{Cu}_{\mathrm{Cd}} \mathrm{Cu}_{i}\right) \cdot+2 e^{\prime} \\
& \left(\mathrm{Cu}_{\mathrm{Cd}} \mathrm{Cu}_{i}\right) \cdot+V_{\mathrm{Cd}}^{\prime \prime}=\left(\mathrm{Cu}_{\mathrm{Cd}}\right)_{2}^{\prime} \\
& \left(\mathrm{Cu}_{\mathrm{Cd}} \mathrm{Cu}_{i}\right)^{\cdot}+2 e^{\prime}+C d_{\mathrm{Cd}}^{x}=\left(\mathrm{Cu}_{\mathrm{Cd}}\right)_{2}^{\prime}+\mathrm{Cd}(g) \\
& \left(\mathrm{Cu}_{\mathrm{Cd}} \mathrm{Cu}_{i}\right)_{2}^{x}+\mathrm{Cd}_{\mathrm{Cd}}^{x}=\left(\mathrm{Cu}_{\mathrm{Cd}} \mathrm{Cu}_{i}\right) \cdot+\left(\mathrm{Cu}_{\mathrm{Cd}}\right)_{2}^{\prime}+\mathrm{Cd}(g) \mid K_{31}=\left[\left(\mathrm{CuCd}_{\mathrm{Cu}_{i}}\right)^{\cdot}\right]\left[\left(\mathrm{Cu}_{\mathrm{Gd}}\right)_{2}^{\prime}\right]\left[\left(\mathrm{Cu}_{\mathrm{Cd}} \mathrm{Cu}_{i}\right)_{2}^{x}\right]^{-1} p_{\mathrm{Cd}} \\
& K_{1}=p_{\mathrm{Cd}} p_{\mathrm{S}_{2}}^{1 / 2} \\
& K_{2}=\left[V_{\mathrm{s}}^{*}\right] n^{2} p_{\mathrm{Cd}}^{-1} \\
& K_{3}=\left[V_{\mathrm{s}}^{* \cdot}\right]\left[V_{\mathrm{Cd}}^{\prime \prime}\right] \\
& K_{4}=n p \\
& K_{5}=\left[V_{\mathrm{s}}^{\cdot}\right] n\left[V_{\mathrm{s}}^{x}\right]^{-1} \\
& K_{6}=\left[V_{\mathrm{s}}^{*}\right] n\left[V_{\mathrm{s}}^{\cdot}\right]^{-1} \\
& K_{7}=\left[V_{\mathrm{Cd}}^{\prime}\right] p\left[V_{\mathrm{Cd}}^{x}\right]^{-1} \\
& K_{8}=\left[V_{\mathrm{Cd}}^{\prime}\right] n\left[V_{\mathrm{Cd}}^{\prime \prime}\right]^{-1} \\
& K_{9}=\left[V_{\mathrm{Cd}}^{x}\right]\left[V_{\mathrm{s}}^{x}\right] \\
& K_{10}=\left[V_{\mathrm{Cd}}^{\prime \prime}\left(\mathrm{Cl}_{\mathrm{S}}^{-}\right)_{2}\right] a_{\mathrm{CdCl}_{2}{ }^{-1}} \\
& K_{11}=\left[\mathrm{Cl}_{\mathrm{S}} \cdot\right] n p \mathrm{CdCl}_{2}^{-1 / 2} p^{-1} \\
& K_{12}=\left[\mathrm{Cl} \cdot{ }_{\mathrm{S}}^{\cdot}\right] n\left[\mathrm{Cl}_{\mathrm{s}}^{x}\right]^{-1} \\
& K_{13}=\left[V_{\mathrm{Cd}}^{\prime \prime} \mathrm{Cl}_{\mathrm{S}}^{\cdot}\right]\left[\mathrm{Cl}_{\mathrm{S}}^{\cdot}\right]^{-1}\left[V_{\mathrm{Cd}}^{\prime \prime}\right]^{-1} \\
& K_{14}=\left[V_{\mathrm{Cd}}^{\prime \prime}\left(\mathrm{Cl}_{\mathrm{S}}^{\cdot}\right)_{2}\right]\left[\mathrm{Cl}_{\mathrm{S}}^{\cdot}\right]^{-1}\left[\mathrm{~V}_{\mathrm{Cd}}^{\prime \prime} \mathrm{Cl}_{\mathrm{S}}^{\cdot}\right]^{-1} \\
& K_{15}=\left[\left(\mathrm{Cu}_{\mathrm{Cd}} \mathrm{Cu}_{i}\right)_{2}^{x}\right] \\
& K_{16}=\left[\left(\mathrm{Cu}_{\mathrm{Cd}} \mathrm{Cu}_{i}\right)^{x}\right]^{1 / 2} p_{\mathrm{Cd}} \\
& K_{17}=\left[\left(\mathrm{Cu}_{\mathrm{Cd}} \mathrm{Cl}_{\mathrm{S}}\right)^{x}\right]^{2} \\
& K_{18}=\left[\mathrm{Cu}_{\mathrm{Cd}^{\prime}}\right]\left[\mathrm{Cu}_{i}^{*}\right]\left[\left(\mathrm{Cu}_{\mathrm{Cd}} \mathrm{Cu}_{i}\right)^{x}\right]^{-1} \\
& K_{19}=\left[\left(\mathrm{Cu}_{\mathrm{Cd}} \mathrm{Cu}_{i}\right)_{2}^{x}\right]\left[\left(\mathrm{Cu}_{\mathrm{Cd}} \mathrm{Cu}_{i}\right)^{x}\right]^{-2} \\
& K_{20}=\left[V_{\mathrm{Cd}}^{\prime \prime}\right]\left[\mathrm{Cu}_{i}^{\cdot}\right]\left[\mathrm{Cu}_{\mathrm{Cd}}^{\prime}\right]^{-1} \\
& K_{21}=\left[\mathrm{Cu}_{\mathrm{Cd}}^{\prime}\right] p\left[\mathrm{Cu}_{\mathrm{Cd}}^{x}\right]^{-1} \\
& K_{22}=\left[\mathrm{Cu}_{i}^{*}\right] n\left[\mathrm{Cu}_{i}^{x}\right]^{-1} \\
& \left.K_{23}=\left[\mathrm{Cu}_{\mathrm{Cd}}^{\prime}\right)_{2}\right]\left[\mathrm{Cu}_{\mathrm{Cd}}^{\prime}\right]^{-2} \\
& K_{24}=\left[\left(\mathrm{Cu}_{\mathrm{Cd}} \mathrm{Cl}_{\mathrm{S}}\right)^{x}\right]\left[\mathrm{Cu}_{\mathrm{Cd}}^{\prime}\right]^{-1}\left[\mathrm{Cl}_{\mathrm{S}}^{-}\right]^{-1}
\end{aligned}
$$

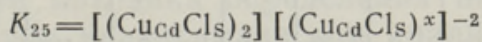

$$
\begin{aligned}
& K_{26}=\left[\mathrm{Cu}_{\mathrm{Cd}}^{\prime} \mathrm{Cl}_{\mathrm{s}}^{\cdot}\right]\left[V_{\mathrm{Cd}}^{\prime \prime} \mathrm{Cl}_{\mathrm{s}}^{-}\right]\left[{V_{\mathrm{Cd}}^{\prime \prime}}^{\prime}\left(\mathrm{Cl}_{\mathrm{s}}^{\cdot}\right)_{2}\right]^{-1}\left[\mathrm{Cu}_{\mathrm{Cd}}^{\prime}\right]^{-1} \\
& K_{27}=\left[\mathrm{Cu}_{\mathrm{Cd}}^{\prime} \mathrm{Cl}_{\mathrm{S}}^{\cdot}\right]\left[\mathrm{Cu}_{i}^{*}\right]^{-1}\left[\mathrm{~V}_{\mathrm{Cd}}^{\prime \prime} \mathrm{Cl}_{\mathrm{s}}^{\cdot}\right]^{-1} \\
& K_{28}=\left[\left(\mathrm{Cu}_{\mathrm{Cd}} \mathrm{Cu}_{i}\right)^{\cdot}\right]^{2} n^{2}\left[\left(\mathrm{Cu}_{\mathrm{Cd}} \mathrm{Cu}_{i}\right)_{2}^{x}\right]^{-1} \\
& K_{29}=\left[\left(\mathrm{Cu}_{\mathrm{Cd}} \mathrm{Cu}_{i}\right) \cdot\right]\left[V_{\mathrm{Cd}}^{\prime \prime}\right]^{-1}\left[\left(\mathrm{Cu}_{\mathrm{Cd}}\right)_{2}^{\prime}\right] \\
& K_{30}=\left[\left(\mathrm{Cu}_{\mathrm{Cd}}\right)_{2}^{\prime}\right] p_{\mathrm{Cd}}\left[\left(\mathrm{Cu}_{\mathrm{Cd}} \mathrm{Cu}_{2}\right)^{\cdot}\right]^{-1} n^{-2}
\end{aligned}
$$

та $\underset{m}{\mathrm{~g}}$ значение $g$ известно; $\gamma_{n}, \alpha_{n}$ и $\beta_{n}$ получают из квазихимических расчетов на основе табл. 1 , в случае $\sigma_{m}$ следует обратиться к [5]. Значения $\gamma_{n}, \alpha_{n}$ и $\beta_{n}$ в броуэровском приближении либо дробные, либо целочисленные, включая и 0 . На практике наиболее вероятны следующие значения $\gamma_{n}, \alpha_{n}, \beta_{n}: \pm \frac{1}{4}, \pm \frac{1}{3}, \pm \frac{1}{2}, 0, \pm 1, \pm 2$. Поэтому целесообразно построить таблицы значений $\gamma_{m g}, \alpha_{m g}, \beta_{m g}$ в зависимо- 
Расчетные значения параметров $\mathbf{g}, \sigma_{m}, \tau_{m}, \gamma_{m g}, \alpha_{m g}$

\begin{tabular}{|c|c|c|c|c|c|c|c|c|c|c|}
\hline \multirow[b]{2}{*}{$X_{m}^{g}$} & \multirow[b]{2}{*}{$g$} & \multirow[b]{2}{*}{$\sigma_{m}$} & \multirow[b]{2}{*}{$\tau_{m}$} & \multicolumn{4}{|c|}{$\gamma_{m g}$} & \multicolumn{3}{|c|}{$\alpha_{m g}$} \\
\hline & & & & $\gamma_{n}=0$ & $\gamma_{n}=\frac{1}{4}$ & $\gamma_{n}=\frac{1}{3}$ & $\gamma_{n}=\frac{1}{2}$ & $\alpha_{n}=0$ & $a_{n}=\frac{1}{4}$ & $\alpha_{n}=\frac{1}{2}$ \\
\hline$e^{\prime}$ & -1 & 0 & 0 & 0 & $\frac{1}{4}$ & $\frac{1}{3}$ & $\frac{1}{2}$ & 0 & $\frac{1}{4}$ & $\frac{1}{2}$ \\
\hline$h$. & 1 & 0 & 0 & 0 & $-\frac{1}{4}$ & $-\frac{1}{3}$ & $-\frac{1}{2}$ & 0 & $-\frac{1}{4}$ & $-\frac{1}{2}$ \\
\hline$V_{\mathrm{s}}^{x}$ & 0 & 1 & 0 & 1 & 1 & 1 & 1 & 0 & 0 & 0 \\
\hline$V_{\mathrm{s}}^{\cdot}$ & 1 & 1 & 0 & 1 & $\frac{3}{4}$ & $\frac{2}{3}$ & $\frac{1}{2}$ & 0 & $-\frac{1}{4}$ & $-\frac{1}{2}$ \\
\hline $\begin{array}{r}V_{\mathrm{s}}^{x} \\
\mathrm{r}\end{array}$ & 2 & 1 & 0 & 1 & $\frac{1}{2}$ & $\frac{1}{3}$ & 0 & 0 & $-\frac{1}{2}$ & -1 \\
\hline$V_{\mathrm{Cd}}^{x}$ & 0 & -1 & 0 & -1 & -1 & -1 & -1 & 0 & 0 & 0 \\
\hline$V_{\mathrm{Cd}}^{\prime}$ & -1 & -1 & 0 & -1 & $-\frac{3}{4}$ & $-\frac{2}{3}$ & $-\frac{1}{2}$ & 0 & $\frac{1}{4}$ & $\frac{1}{2}$ \\
\hline$V_{\mathrm{Cd}}^{\prime \prime}$ & -2 & -1 & 0 & -1 & $-\frac{1}{2}$ & $-\frac{1}{3}$ & 0 & 0 & $\frac{1}{2}$ & 1 \\
\hline $\mathrm{Cl}_{\mathrm{S}}^{x}$ & 0 & $\frac{1}{2}$ & $\frac{1}{2}$ & $\frac{1}{2}$ & $\frac{1}{2}$ & $\frac{1}{2}$ & $\frac{1}{2}$ & $\frac{1}{2}$ & $\frac{1}{2}$ & $\frac{1}{2}$ \\
\hline $\mathrm{Cl}_{\mathrm{s}}$ & 1 & $\frac{1}{2}$ & $\frac{1}{2}$ & $\frac{1}{2}$ & $\frac{1}{4}$ & $\frac{5}{6}$ & 0 & $\frac{1}{2}$ & $\frac{1}{4}$ & 0 \\
\hline $\mathrm{Cu}_{\mathrm{Cd}}^{x}$ & 0 & $-\frac{1}{2}$ & $-\frac{1}{2}$ & $-\frac{1}{2}$ & $-\frac{1}{2}$ & $-\frac{1}{2}$ & $-\frac{1}{2}$ & $-\frac{1}{2}$ & $-\frac{1}{2}$ & $-\frac{1}{2}$ \\
\hline $\mathrm{Cu}_{\mathrm{Cd}}^{\prime}$ & -1 & $-\frac{1}{2}$ & $-\frac{1}{2}$ & $-\frac{1}{2}$ & $-\frac{1}{4}$ & $-\frac{1}{6}$ & 0 & $-\frac{1}{2}$ & $-\frac{1}{4}$ & 0 \\
\hline $\mathrm{Cu}_{i}^{x}$ & 0 & $\frac{1}{2}$ & $-\frac{1}{2}$ & $\frac{1}{2}$ & $\frac{1}{2}$ & $\frac{1}{2}$ & $\frac{1}{2}$ & $-\frac{1}{2}$ & $-\frac{1}{2}$ & $-\frac{1}{2}$ \\
\hline $\mathrm{Cu}_{i}^{*}$ & 1 & $\frac{1}{2}$ & $-\frac{1}{2}$ & $\frac{1}{2}$ & $\frac{1}{4}$ & $\frac{5}{6}$ & 0 & $-\frac{1}{2}$ & $-\frac{3}{4}$ & -1 \\
\hline$V_{\mathrm{Cd}}^{\prime} \mathrm{Cl}_{\mathrm{s}}$ & 0 & $-\frac{1}{2}$ & $\frac{1}{2}$ & $-\frac{1}{2}$ & $-\frac{1}{2}$ & $-\frac{1}{2}$ & $-\frac{1}{2}$ & $\frac{1}{2}$ & $\frac{1}{2}$ & $\frac{1}{2}$ \\
\hline$V_{\mathrm{Cd}}^{\prime \prime} \mathrm{Cl}_{\mathrm{s}}$ & -1 & $-\frac{1}{2}$ & $\frac{1}{2}$ & $-\frac{1}{2}$ & $-\frac{1}{4}$ & $-\frac{1}{6}$ & 0 & $\frac{1}{2}$ & $\frac{3}{4}$ & 1 \\
\hline$\left(V_{\mathrm{Cd}}^{\prime \prime}\right)\left(\mathrm{Cl}_{\mathrm{s}}\right)_{2}$ & 0 & 0 & 1 & 0 & $\begin{array}{l}0 \\
1\end{array}$ & $\begin{array}{l}0 \\
1\end{array}$ & $\begin{array}{l}0 \\
1\end{array}$ & 1 & $\begin{array}{l}1 \\
3\end{array}$ & $\begin{array}{l}1 \\
1\end{array}$ \\
\hline$V_{\mathrm{Cd}}^{\prime}\left(\mathrm{Cl}_{\mathrm{s}}^{-}\right)_{2}$ & 1 & 0 & 1 & 0 & $-\frac{1}{4}$ & $-\frac{1}{3}$ & $-\frac{1}{2}$ & 1 & $\frac{5}{4}$ & $\frac{\pi}{2}$ \\
\hline $\mathrm{Cu}_{\mathrm{Cd}}^{\prime} \mathrm{Cu}_{i}$ & 0 & 0 & -1 & 0 & $\begin{array}{r}0 \\
-\quad 1\end{array}$ & $\begin{array}{l}0 \\
1\end{array}$ & $\begin{array}{l}0 \\
1\end{array}$ & -1 & $\begin{array}{r}-1 \\
5\end{array}$ & $\begin{array}{r}-1 \\
3\end{array}$ \\
\hline $\mathrm{Cu}_{\mathrm{Cd}}^{x} \mathrm{Cu}_{i}$ & 1 & 0 & -1 & 0 & $-\frac{1}{4}$ & $-\frac{1}{3}$ & $-\frac{1}{2}$ & -1 & $-\frac{\pi}{4}$ & $-\frac{\pi}{2}$ \\
\hline $\mathrm{Cu}_{\mathrm{Cd}}^{\prime} \mathrm{Cl}_{\mathrm{s}}^{-}$ & 0 & 0 & 0 & 0 & 0 & 0 & 0 & 0 & 0 & 0 \\
\hline$\left(\mathrm{Cu}_{\mathrm{Cd}}\right)_{2}^{\prime}$ & -1 & -1 & -1 & -1 & $-\frac{3}{4}$ & $-\frac{2}{3}$ & $-\frac{1}{2}$ & -1 & $-\frac{3}{4}$ & $-\frac{1}{2}$ \\
\hline$\left(\mathrm{Cu}_{\mathrm{Cd}}\right)_{2}^{\prime \prime}$ & -2 & -1 & -1 & -1 & $-\frac{1}{2}$ & $-\frac{1}{3}$ & 0 & -1 & $-\frac{1}{2}$ & 0 \\
\hline
\end{tabular}

сти от различных значений экспериментальных параметров $\gamma_{n}, \alpha_{n}, \beta_{n}$ (типа табл. 2) и выбирать оттуда значения $\gamma_{m g}, \alpha_{m g}, \beta_{m g}$ из тсй колонки, которая соответствует экспериментальным значениям $\gamma_{n}, \alpha_{n}, \beta_{n}$. Таким образом, в отличие от метода Крегера-Броуэра [ํ] отпадает 
необходимость заранее выбнрать приближенное УЭН, что вносит элемент неопределенности в квазихимический анализ дефектов. Доминирующие заряженные дефекты определяются из табл. 2 среди положительно $(+)$ и отрицательно $(-)$ заряженных дефектов, имеющих одинаковые значения квазихимических параметров

$$
\begin{aligned}
& \gamma_{(+)}=\gamma_{(-)}, \\
& \alpha_{(+)}=\alpha_{(-)}, \\
& \beta_{(+)}=\beta_{(-)} .
\end{aligned}
$$

Доминирующие примесные дефекты определяются из условия $\mathrm{Gmg}=1$ (Cl) нли $\beta_{m g}=1$ (Cu), так как их концентрации практически равны $[\mathrm{Cl}]$ и $[\mathrm{Cu}]$ соответственно.

Из табл. 2 следует, что для собственных дефектов

$$
\tau_{m}=\vartheta_{m}=0 .
$$

В силу (15) нейтральные собственные дефекты $(g=0)$ имеют

$$
\alpha_{m g}=\beta_{m g}=0,
$$

т. е. их концентрации не зависят от уровня легирования. Относительно $\gamma_{m g}, \alpha_{m g}, \beta_{m g}$ из табл. 2 вытекают следующие закономерности: а) однотипные дефекты, различающиеся только зарядом, имеют одинаковые показатели степени $\gamma_{m g}=\sigma_{m}, \alpha_{m g}=\tau_{m}, \beta_{m g}=\theta_{m}$, если $\gamma_{n}=\alpha_{n}=\beta_{n}=0$, т. е. в том случае, если $n$ не зависит от $p_{\text {Cd, }}$ [Cl] и [Cu]. Отсюда следует, что общая концентрация однотипных дефектов $X_{m}$ имеет степенную зависимость от $p_{\mathrm{Cd}},[\mathrm{Cl}],[\mathrm{Cu}]$ вида

$$
\left[X_{m}\right] \sim p_{\mathrm{Cd}}^{\gamma_{m}}[\mathrm{Cl}]^{\alpha_{m}}[\mathrm{Cu}]^{\beta_{m}}
$$

б) $\gamma_{m g}, \alpha_{m g}, \beta_{m g}$ нейтральных дефектов не зависят от $\gamma_{n}, \alpha_{n}, \beta_{n}$.

Учитывая экспоненциальную зависимость $K_{m}^{g}=K_{m 0}^{g} \exp \left(-\frac{\Delta H_{m g}}{k T}\right)$,

получаем корреляционную связь между препаративными условиями и дефектным составом в пределах одной броуэровской области в виде

$$
\left[X_{m}^{g}\right]=K_{m 0}^{g} p_{\mathrm{Cd}}^{\gamma_{m g}}[\mathrm{Cl}]^{\alpha_{m !}}[\mathrm{Cu}]^{\beta_{m g}} \exp \left(-\frac{\Delta H_{m g}}{k T}\right) .
$$

Описанная в настоящей работе методика определения параметров точечных дефектов использована при квазихимическом анализе систем $\mathrm{CdS}: \mathrm{Cl}\left[{ }^{14}\right]$ и $\mathrm{CdS}: \mathrm{Cl}: \mathrm{Cu}\left[{ }^{15}\right]$.

Соотношение (18) сохраняется и для «замороженных» дефектов в «мгновенно» охлажденных до низких температур образцах. Это важное обстоятельство использовано при создании люминесцентных методов квазихимического анализа низкотемпературного дефектного состава широкозонных полупроводников, базирующихся на степенной зависи мости интенсивности свечения от концентрации оптически активных центров [16-19].

\section{Л И ТЕ РА Т У Р А}

1. Точечные дефекты в твердых телах. М., 1979.

2. Кукк П. Л. Основы направленного дефектообразования в полупроводниковых соединениях. - Ж. неорган. хим., 1984, 29, № 6, 1365-1373.

3. Кукк П. Л. Формирование оптически активных центров в полупроводниках $\mathrm{A}^{\mathrm{II}} \mathrm{B}^{\mathrm{VI}}$. - В кн.: Физика и техническое применение полупроводников $\mathrm{A}^{\mathrm{II}} \mathrm{B}^{\mathrm{VI}}$, 1. Вильнюс, $1983,74-75$. 
4. Кукк П. Л. Направленное дефектообразование в широкозонных полупроводниках. - В кн.: Физико-химические основы методов получения и исследования материалов электронной техники. Новосибирск, 1984, 45-47.

5. Kukk, P. L., Aarna, H. A., Voogne, M. P. High-temperature point defects in CdS:Cu. - Phys. Stat. Sol.(a), 1981, 63, N 2, 389-399.

6. Kukk, P. L., Aarna, H. A. Solubility of Ag in CdS single crystals. - Phys. Stat. Sol.(a), 1982, 69, N 2, K109-K112.

7. Бьюб Р. Фотопроводимость твердых тел. М., 1962.

8. Гурвич А. М. Введение в физическую химию кристаллофосфоров. М., 1982.

9. Крегер Ф. Химия несовершенных кристаллов. М., 1969.

10. Vydyanath, $H$. R., Kröger, $F$. A. The defect structure of silver doped CdS. J. Phys. Chem. Solids, 1975, 36, 509-520.

11. Ray, A. K., Kröger, F. A. The defect structure of pure and doped $\mathrm{ZnSe}$ - J. Electrochem. Soc., 1978, 125, N 8, 1348-1355.

12. Ray, A. K., Kröger, F. A. The defect structure of ZnSe:Ga. - J. Electrochem. Soc., 1978, 125, N 8, 1355-1361.

13. Woodbury, H. H. Defect characterization: diffusivity and electrical measurements. - In: II-VI Semiconducting Compounds. New York, Amsterdam, 1967, 244276.

14. Алтосаар М. Э., Кукк П. Л., Хийе Я. В. Растворимость хлора в CdS и структура точечных дефектов в CdS:Cl. - Ж. неорган. хим., 1983, 23, № 1, 69-75.

15. Kukk, P. L., Altosaar, M. E. Defect structure of Cl- and Cu-doped CdS heat treated in $\mathrm{Cd}$ and $\mathrm{S}_{2}$ vapor. - J. Solid State Chem., 1983, 43, N 1, 1-10.

16. Kukk, P. L., Erm, A.J. The structure of radiative and nonradiative recombination centres in activated CdS phosphors. - Phys. Stat. Sol.(a), 1981, 67, N 2, $395-406$.

17. Кукк П. Л. Люминесцентный анализ точечных дефектов в легированном сульфнде кадмия. - Укр. физ. Ж., 1982, 27, № 5, 754-761.

18. Kukk, P. L., Palmre, O. V., Mellikov, E. J. The structure of recombination centres in activated ZnSe phosphors. - Phys. Stat. Sol.(a), 1982, 70, N 1, 35-42.

19. Кукк П. Л., Крусток Ю. И., Эрм А. Я., Мядассон Я. Э., Моин М. Д. Структура точечных дефектов в примесном сульфиде цинка. - Ж. неорган. хим., 1984, 29, № 1, 46-50.

Таллинский политехнический институт

Поступила в редакцию $11 /$ III 1985

\section{P. KUKK, H. AARNA, Mare ALTOSAAR, \\ J. MÄDASSON, E. MELLIKOV \\ PUNKTDEFEKTIDE KVAASIKEEMILISTE PARAMEETRITE MÄARAMINE POOLJUHTUHENDITES}

On arendatud uus metoodika punktdefektide kvaasikeemiliste parameetrite määramiseks muutuva koostisega pooljuhtühendite tahkes faasis. Lähtudes vabade laengukandjate kōrgetemperatuuriliste mõōtmiste tulemustest ja nimetatud parameetrites sisalduvatest konstantidest, on vōimalik määrata punktdefektide kontsentratsioonide astmenäitajad sōltuvana käsitlustingimustest. Esitatud metoodika ei nõua elektroneutraalsuse tingimuse lähenduse etteandmist ja on rakendatav nii legeerimata kui ka mitme lisandiga legeeritud pooljuhtühendite puhul.

\section{P. KUKK, H. AARNA, Mare ALTOSAAR, \\ J. MADASSON, E. MELLIKOV}

\section{DETERMINATION OF QUASICHEMICAL PARAMETERS OF POINT DEFECTS IN SEMICONDUCTING COMPOUNDS}

A new method is developed for determining quasichemical parameters of point defects in semiconducting solid phases - the exponents of point defect dependences on treatment conditions, proceeding from high-temperature free charge carrier concentration measurements and constants used in quasichemical calculations that are included in these parameters. The proposed method does not need to assign any approximation of electroneutrality conditions and may be used in case of both undoped and complexly doped semiconducting compounds. 